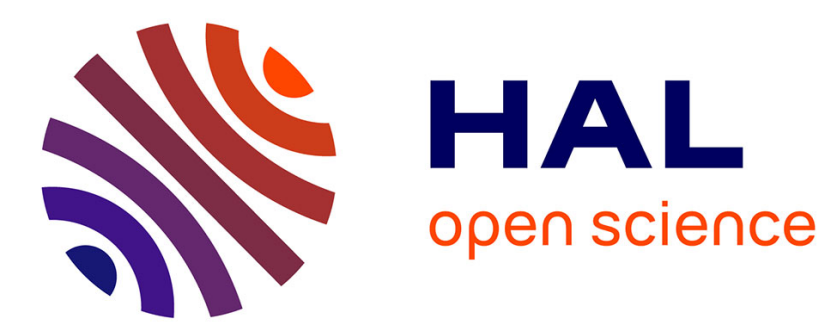

\title{
Controlling quantum dynamics regardless of laser beam spatial profile and molecular orientation
}

Herschel Rabitz, Gabriel Turinici

\section{To cite this version:}

Herschel Rabitz, Gabriel Turinici. Controlling quantum dynamics regardless of laser beam spatial profile and molecular orientation. Physical Review A: Atomic, molecular, and optical physics [19902015], 2007, 75 (4), pp.043409. hal-00536535

\section{HAL Id: hal-00536535 \\ https://hal.science/hal-00536535}

Submitted on 9 Apr 2013

HAL is a multi-disciplinary open access archive for the deposit and dissemination of scientific research documents, whether they are published or not. The documents may come from teaching and research institutions in France or abroad, or from public or private research centers.
L'archive ouverte pluridisciplinaire HAL, est destinée au dépôt et à la diffusion de documents scientifiques de niveau recherche, publiés ou non, émanant des établissements d'enseignement et de recherche français ou étrangers, des laboratoires publics ou privés. 


\title{
Controlling Quantum Dynamics Regardless of Laser Beam Spatial Profile and Molecular Orientation
}

\author{
Herschel Rabitz* \\ Department of Chemistry, Princeton University, Princeton, NJ 08544-1009, USA \\ Gabriel Turinici ${ }^{\dagger}$ \\ CEREMADE, Universite Paris Dauphine, \\ Place du Marechal De Lattre De Tassigny, 75775 PARIS Cedex 16, France
}

(Dated: March 13, 2007)

\begin{abstract}
In a typical experiment aiming to control quantum dynamics phenomena each molecule experiences the same temporal laser field, but with an amplitude that depends on the spatial location and orientation of the molecule in the laser beam. It is proved under commonly arising conditions that at least one optimal laser field exists which will control all molecules in the sample, regardless of their orientation or spatial location. The optimal laser field may consist of a multipolarization control containing up to three orthogonal independently shaped components. The analysis also includes the prospect of multi-partite control where the field couples distinct groupings of states (e.g., multiple vibronic states), but without direct coupling within a group of states. This conclusion shows that achieving quantum control is not a matter of striking a compromise over the sample diversity, but rather a task subject to optimization to reach the highest possible level of control for all molecules in the sample.
\end{abstract}

PACS numbers: $32.80 . \mathrm{Qk}$

Keywords: quantum control, controllability, bilinear system, laser profile

*Electronic address: hrabitz@princeton.edu

${ }^{\dagger}$ Electronic address: Gabriel.Turinici@dauphine.fr 
The control of quantum phenomena is being increasingly explored, both theoretically [14] and in the laboratory [5-9]. An important goal is to explain the growing number of successful experiments as a foundation for understanding what may be expected for the future of the field. A recent paper identified one key factor as the simplicity of the quantum control search landscape permitting ready access to high quality solutions [10]. This paper lays out a second critical factor as the ease of achieving control over a full sample of molecules, despite the presence of spatial inhomogeneous effects and random molecular orientations.

Optimally shaped laser pulses currently can have temporal structure on the order of femtoseconds and total pulse lengths of a picosecond or less. The samples under control are often optically thin and the pulses sufficiently intense such that back action to reshape the pulses in the medium can be ignored. However, the pulses have spatial profiles (e.g., a Gaussian) transverse to the direction of propagation. The beam is frequently also focused down to have a narrow waist in a plane at some point along the direction of propagation, with the beam fanning out before and after the focal plane. Thus, a molecule in one spatial location will experience a distinct field amplitude from another molecule in a different location. This situation can be described by each molecule being exposed to the same temporal laser field multiplied by an overall factor depending on the spatial location of the molecule in the laser beam. Compounding this situation is the fact that the molecules in the sample will generally be randomly oriented. A basic question is whether a single temporal laser pulse shape can control all of the molecules, regardless of their spatial location or orientation. Previous optimal control simulations $[11,12]$ hinted that the answer is positive. Based on recent theoretical advances in assessing quantum controllability, this paper addresses this question to reveal that full control can be achieved regardless of spatial location or molecular orientation including with multiple polarized and shaped laser pulses.

Consider a collection of identical molecules of internal Hamiltonian $H_{0}$ subjected to external control from laser pulses of temporal structure $\epsilon^{s}(t), s=1, ., S$ and linear polarization directions $\widehat{\epsilon^{s}}$ that couple to the molecules through a dipole moment vector operator $\mu^{s}$. Here $S$ can be 1,2 or 3 corresponding to there being up to three mutually perpendicular polarized control pulses. We denote by $x$ the internal coordinates of each molecule and the goal is to achieve control over some aspect of the internal motion. The propagating laser pulse has the spatial profile $u^{s}(R)$ with $R$ denoting a location in the sample within a laboratory based frame (see Figure 1). The Figure shows a single polarized pulse (i.e., $s=1$ ) propagating, 
and a second polarization $s=2$ rotated $90^{\circ}$ could propagate as well in the same direction. If a third shaped polarization control $s=3$ was used [4] it would propagate perpendicular to the configuration in the Figure (see also [13]). Typically, $u^{s}(R)$ may have a Gaussian like shape or some other form [11]. The $s$-th control field seen by a molecule at location $R$ is $u^{s}(R) \cos \left(\alpha_{R}^{s}\right) \epsilon^{s}(t)$ where $u^{s}(R)$ acts as a constant amplitude factor for any value of $R$, and $\alpha_{R}^{s}$ (depending on $R$ ) is the angle between the pulse polarization unit vector $\widehat{\epsilon^{s}}$ and the molecular dipole vector $\mu^{s}$. Additionally, the spatial dependence of $u(R)$ may also contain a phase factor which can result in $u^{s}(R)$ changing sign at certain locations $R$. See also Remark 2 below. The wavefunction $\Psi(t, x ; R)$ of the molecule at $R$ and orientation $\alpha_{R}^{s}$ obeys the Schrödinger equation:

$$
\begin{aligned}
& i \hbar \frac{\partial}{\partial t} \Psi(t, x ; R)=\left(H_{0}(x)-\sum_{s=1}^{S} u^{s}(R) \cos \left(\alpha_{R}^{s}\right) \mu^{s}(x) \epsilon^{s}(t)\right) \Psi(t, x ; R) \\
& \Psi\left(t_{0}, x ; R\right)=\Psi^{0}(x ; R) .
\end{aligned}
$$

In this analysis the pulse time scale is assumed to be short compared to the molecular rotational periods, as expected for large molecules and in the condensed phases. Thus, the coordinates $R$ act as parameters characterizing each molecule's wavefunction under the action of the control. The analysis below may also be extended to consider the density matrix.

The question is whether it is possible to find a set of pulse shapes $\left(\epsilon^{s}(t)\right)_{s=1}^{S}$ capable of driving the collection of molecules in the entire sample to a common target, despite the fact that molecules at $R$ and $R^{\prime}$ may experience distinct amplitudes $u^{s}(R) \cos \left(\alpha_{R}^{s}\right) \epsilon^{s}(t)$ and $u^{s}\left(R^{\prime}\right) \cos \left(\alpha_{R}^{s \prime}\right) \epsilon^{s}(t)$ with $u^{s}(R) \cos \left(\alpha_{R}^{s}\right) \neq u^{s}\left(R^{\prime}\right) \cos \left(\alpha_{R}^{s \prime}\right)$ ? This question can be understood as a controllability issue. The assessment of whether a single shaped laser pulse can drive independent (i.e., distinct) quantum systems to their respective target states was addressed theoretically [14] and applied to the optimal dynamic discrimination of separate quantum systems [15]. To treat the particular case of identical molecules, we proved in [16] a theoretical result for $S=1$ whose generalization will be given below.

Before stating the key result in this work, $H_{0}$ is first represented in its eigenbasis $\phi_{1}, \ldots$, $\phi_{N}$ as $H_{0}=\operatorname{diag}\left\{\lambda_{1}, \ldots, \lambda_{N}\right\}$ and $\omega_{a b}$ denotes the transition frequencies $\omega_{a b}=\lambda_{a}-\lambda_{b}$. We construct for each coupling dipole $\mu^{s}$ the connectivity graph [17] by drawing an edge between any two states coupled by some dipole $\mu^{s}: G^{s}=\left(V, E^{s}\right), V=\{1, \ldots, N\}, E^{s}=$ $\left\{(i, j) ;\left\langle\phi_{i}\left|\mu^{s}\right| \phi_{j}\right\rangle \neq 0\right\}$. We also introduce the total graph $G=\left(V, \cup_{s=1}^{S} E^{s}\right)$. A graph $G^{s}$ is 


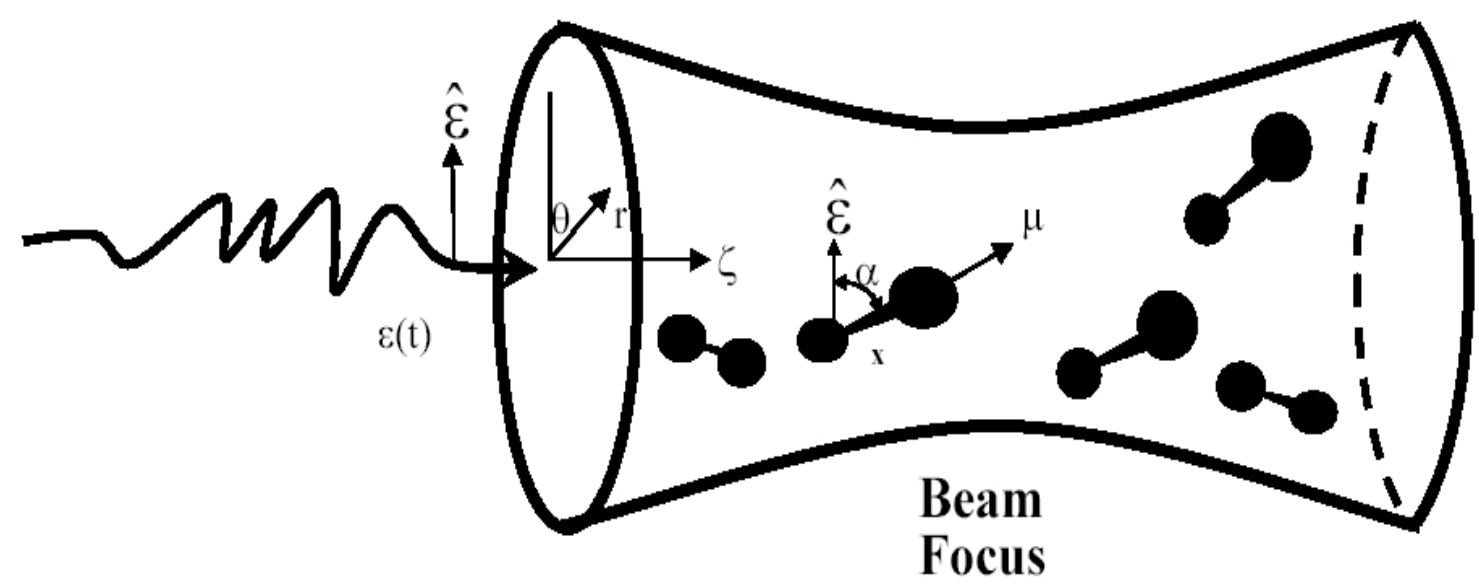

FIG. 1: The shaped laser pulse $\epsilon(t) \hat{\epsilon}$ (i.e., linearly polarized for illustration with the superscript label $s=1$ implicitly understood; see the text for a discussion of the generalized situation with up to two additional polarized control fields, $s=2$ and 3) control propagates with a spatial profile $u(R)=u(r, \theta, \zeta)$ dependent on the location $R$ with $R=(r, \theta, \zeta)$ being cylindrical coordinates, where $(r, \theta)$ denotes the radial $r \geq 0$ and angular $0 \leq \theta \leq 2 \pi$ variables in the plane perpendicular to the direction of propagation and $\zeta$ is the coordinate along the axis of propagation. A molecule with internal coordinates $x$ at location $R$ and orientation $\alpha$ between the dipole $\mu$ and the pulse polarization direction $\hat{\epsilon}$ will experience a control of amplitude $u(R) \cos \left(\alpha_{R}\right)$. Despite the spatial and orientational effects, the internal dynamics of all molecules (depicted here as diatomics for illustrative simplicity) in the volume can be fully controlled, if one molecule is controllable anywhere.

said to be bipartite if the set of states $V$ can be partitioned into two disjoint sets $V_{1}$ and $V_{2}\left(V_{1} \cup V_{2}=V, V_{1} \cap V_{2}=\emptyset\right)$ such that all edges are between one element in $V_{1}$ and one element in $V_{2}: E^{s} \subset V_{1} \times V_{2}$. The same definition applies for $G$ (see Figure 2).

The bipartite nature of a graph is often encountered in the laser manipulation of matter (e.g., when lasers couple some states on a "ground" state surface with states on some "higher" electronic excited state surface, but do not couple states from the same surface [3]). The prior work [16] did not treat such common bipartite situations. The present analysis will allow for identifying the compatibility conditions that ensure positive results under these circumstances.

A preliminary condition for the controllability of the full collection of molecules with a 


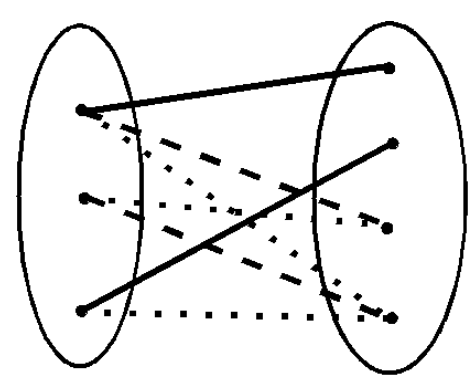

FIG. 2: An example of a total bipartite graph $G$. The partition of vertices into two classes (left and right) is such that no edges exist between vertices of the same class. Different classes of edges (solid, dotted and dashed) correspond to the $S=3$ distinct polarized laser pulses. All graphs $G^{1}, G^{2}$ and $G^{3}$ are bipartite as well.

single set of laser fields $\left(\epsilon^{s}(t)\right)_{s=1}^{S}$ is that the laser pulses $u^{s}(R) \cos \left(\alpha_{R}^{s}\right) \epsilon^{s}(t), s=1, ., S$ are capable of controlling a molecule at least at one location $R$ in the profile.

Theorem. Consider a characteristic sampling of $M$ molecules located at $R_{1}, \ldots, R_{M}$ with dynamics described by Eqn. (1), none with dipole components orthogonal to any of the laser polarization directions: $u^{s}\left(R_{i}\right) \cos \left(\alpha_{R_{i}}^{s}\right) \neq 0$, for all $s \leq S, i \leq M$. Suppose that at least one molecule is controllable. To avoid trivial settings we consider that controllability does not hold if one laser is omitted. Also suppose that

$$
\omega_{a b} \neq \omega_{a^{\prime} b^{\prime}}, \text { for all }(a, b) \neq\left(a^{\prime}, b^{\prime}\right)
$$

1. If at least one of the following hypothesis (3) or (4) is true,

None of the connectivity graphs $G^{s}=\left(V, E^{s}\right), s=1, ., S$ is bipartite.

Any two molecules are distinguishable

i.e., for any $i<j \leq M$ there exists $s \leq S$ such that

$0 \neq\left|u^{s}\left(R_{i}\right) \cos \left(\alpha_{R_{i}}^{s}\right)\right| \neq\left|u^{s}\left(R_{j}\right) \cos \left(\alpha_{R_{j}}^{s}\right)\right| \neq 0$,

then the full collection of molecules is controllable implying that a laser pulse $\left(\epsilon^{s}(t)\right)_{s=1}^{S}$ exists such that the ensemble starting from $\Psi^{f}\left(t^{0}, x ; R_{k}\right)_{k=1}^{M}=\Psi^{0}(x)$ and propagating under the Schrödinger equation (1) reaches $\Psi^{f}\left(t^{f}, x ; R_{k}\right)_{k=1}^{M}=\Psi^{f}(x)$ at some time $t^{f}$.

2. Let $S=1$. Consider that the hypothesis (4) is not verified and the connectivity graph $G$ of the system is bipartite $V=V_{1} \cup V_{2}, E \subset V_{1} \times V_{2}$. In this case a laser pulse $\epsilon(t)$ 
exists such that the ensemble starting from $\Psi^{f}\left(t^{0}, x ; R_{k}\right)_{k=1}^{M}=\Psi^{0}(x)$ and propagating under the Schrödinger equation (1) reaches $\Psi^{f}\left(t^{f}, x ; R_{k}\right)_{k=1}^{M}=\Psi^{f}(x)$ at some time $t^{f}$ provided that we do not require moving population between the bipartite components

$$
\sum_{\phi \in V_{1}}\left|\left\langle\Psi^{0} \mid \phi\right\rangle\right|^{2}=\sum_{\phi \in V_{1}}\left|\left\langle\Psi^{f} \mid \phi\right\rangle\right|^{2}
$$

Proof of the Theorem Without loss of generality we may assume that $H_{0}$ and $\mu^{s}$ are traceless. We also denote by $\operatorname{diag}\left(d_{1}, \ldots, d_{P}\right)$ the matrix obtained by setting the square matrices $d_{1}, \ldots, d_{P}$ on its diagonal [16]. This definition allows for introducing $\mathcal{H}_{0}=\operatorname{diag}\left(H_{0}, \ldots, H_{0}\right)$, as a $M N \times M N$ matrix constructed from $M$ replicas of $H_{0}$ and $\mathcal{B}^{s}=\operatorname{diag}\left(u_{1}^{s} \mu^{s}, \ldots, u_{M}^{s} \mu^{s}\right)$ for $u_{k}^{s}=u^{s}\left(R_{k}\right) \cos \left(\alpha_{R_{k}}^{s}\right), 1 \leq k \leq M$. By assembling the $M$ Schrödinger equations (1), the evolution of the set of molecules can be written as a system with block diagonal entries:

$$
i \hbar \frac{\partial}{\partial t}|\Psi(t)\rangle=\left(\mathcal{H}_{0}-\sum_{s=1}^{S} \mathcal{B}^{s} \epsilon^{s}(t)\right)|\Psi(t)\rangle
$$

Denote by $\mathcal{L}$ the Lie algebra generated by the matrices $i \mathcal{H}_{0}$ and $i \mathcal{B}^{s}, s=1, ., S$. We index by $\xi \in \Xi$ (of cardinality $K$ ) all pairs $\xi=(a, b), a<b$ with $\mu_{\xi=(a, b)}^{s} \neq 0$ at least for some $s \leq S$. We also denote $\xi^{\dagger}=(b, a)$. For each $s \leq S$ we compute $a d_{i \mathcal{H}_{0}}^{\ell} i \mathcal{B}^{s}=\left[i \mathcal{H}_{0}, \ldots,\left[i \mathcal{H}_{0}, i \mathcal{B}^{s}\right] \ldots\right]$ with the iterative commutators taken $\ell=1, \ldots, 2 K$ times. We show as in [16] that under the hypothesis (2) all elements $\left\{\operatorname{diag}\left(u_{1}^{s} S_{\xi}, \ldots, u_{M}^{s} S_{\xi}\right), \operatorname{diag}\left(u_{1}^{s} Y_{\xi}, \ldots, u_{M}^{s} Y_{\xi}\right) ; \mu_{\xi}^{s} \neq 0, s=1, ., S, \xi \in\right.$ $\Xi\}$ are obtained. The commutators built from these elements will also be computed. When either hypothesis (3) or (4) is verified the algebra $\oplus_{k=1}^{M} s u(N)$ is obtained. The connecting path approach [16] needs to be modified by the presence of differently polarized lasers. The existence of such a path is a non-trivial consequence of the hypotheses (3) and (4), which generalizes the controllability condition in [16] for $S>1$. We refer the reader to the Appendix for further details.

Consider now $S=1$ (we drop all indices $s$ ) and the circumstance that for some $i<$ $j \leq M, u_{i}=-u_{j}$ and place all such $j$ at the end of the list: $u_{1}, \ldots, u_{T}, u_{T+1}, \ldots, u_{U}, u_{U+1}=$ $-u_{1}, \ldots, u_{M}=-u_{T}(U \geq T, U+T=M)$. When the total graph $G$ is bipartite we obtain the following structure for the matrices $H_{0}$ and $\mu: H_{0}=\left(\begin{array}{cc}H_{0}^{1} & 0 \\ 0 & H_{0}^{2}\end{array}\right), \mu=\left(\begin{array}{cc}0 & B \\ B^{*} & 0\end{array}\right)$. Define

$$
\begin{aligned}
& \mathcal{L}_{U}=\left\{\operatorname{diag}\left\{X_{1}, \ldots, X_{M}\right\}, X_{p} \in \operatorname{su}(N), p \leq U ;\right. \\
& \left.X_{U+k}=\left(\begin{array}{cc}
X_{k}^{1} & -X_{k}^{\mu} \\
\left(X_{k}^{\mu}\right)^{\dagger} & X_{k}^{2}
\end{array}\right) \text { if } X_{k}=\left(\begin{array}{cc}
X_{k}^{1} & X_{k}^{\mu} \\
-\left(X_{k}^{\mu}\right)^{\dagger} & X_{k}^{2}
\end{array}\right)\right\},
\end{aligned}
$$


(here ${ }^{\dagger}$ represents transpose conjugation). Note that $i \mathcal{H}_{0}$ and $i \mathcal{B}$ belong to $\mathcal{L}_{U}$ and that the set $\mathcal{L}_{U}$ is an algebra, i.e., it is closed to commutation. This result shows that $\mathcal{L}=\mathcal{L}_{U}$. The matrix corresponding to the $(U+k)$-th system is thus completely determined by the matrix of the $k$-th system. In particular, $\mathcal{L}$ is isomorphic to $\oplus_{k=1}^{U} s u(N)$. Using a result from [18] we conclude that starting from $\Psi^{f}\left(t^{0}, x ; R_{k}\right)_{k=1}^{M}=\Psi^{0}(x)$ one can reach any $\Psi^{f}\left(t^{f}, x ; R_{k}\right)_{k=1}^{M}=$ $\left(e^{X_{k}} \Psi^{0}(x)\right)_{k=1}^{M}$ at some time $t^{f}$, with $X=\operatorname{diag}\left\{X_{1}, \ldots, X_{M}\right\} \in \mathcal{L}_{U}$.

Let $\Psi_{j}^{0}$ be the components of $\Psi^{0}(x)$ over the set $\operatorname{span}\left(V_{j}\right), j=1,2$ and similarly $\Psi_{j}^{f}$ be the components of $\Psi^{f}(x)$ over $\operatorname{span}\left(V_{j}\right), j=1,2$. Under the compatibility condition (5) : $\left\|\Psi_{j}^{0}\right\|^{2}=\left\|\Psi_{j}^{f}\right\|^{2}, j=1,2$. Thus, for any $k \leq T$ one can find antihermitian matrices $X_{k}^{1}$ and $X_{k}^{2}$ with $\Psi_{1}^{f}=e^{X_{k}^{1}} \Psi_{1}^{0}$ and $\Psi_{2}^{f}=e^{X_{k}^{2}} \Psi_{2}^{0}$. It follows that $\Psi^{f}=e^{\operatorname{diag}\left\{X_{k}^{1}, X_{k}^{2}\right\}} \Psi^{0}$, q.e.d.

The Theorem implies that at least one laser pulse exists which can simultaneously steer all molecules in the sample to the desired final state from a common initial condition (regardless of their spatial location and orientation), with the only caveat that finite pulse energy will ultimately limit the extent of the sample volume where control can be achieved. As the pulse amplitude is locally specified by an overall multiplicative factor $u^{s}(R) \cos \left(\alpha_{R}^{s}\right)$ that depends on the spatial coordinates and local orientation of the molecules within the sample, it follows that different quantum dynamical pathways will be taken by molecules at distinct spatial locations and orientations before they all eventually reach the same common target at the final time $t_{f}$ (i.e., molecules at distinct locations and orientations will reach the same target state by different mechanisms). This analysis explains the success of simulations reported earlier $[11,16]$ and, most importantly, provides the basis to understand how the molecules in a finite volume transcending different laboratory environments (i.e., locations and molecular orientations) can all be swept to the target by a suitable optimal pulse. If the theorem was not true, then at best only a small subset of molecules could be fully controlled within the sample. However, applying a non-optimized pulse will likely result in control of each molecule in the sample to a different degree, depending on its location and orientation. Remark 1 The proof of the Theorem also implies that a field $\epsilon^{s}(t), s=1, ., S$ exists which will permit control over any subset of molecules that have a specific combined spatial location and orientation such that $u^{s}(R) \cos \left(\alpha_{R}^{s}\right), s=1, ., S$ has a constant value $\mathcal{A}^{s}, s=1, ., S$. A collection of equivalence classes of molecules is defined with each class having a specific set of values of $\mathcal{A}^{s}$. In contrast to the objective in the Theorem, in some circumstances the goal may shift to controlling those molecules just belonging to one such class. In the laboratory 
this outcome would call for a feedback signal sensitive to spatial location and molecular orientation.

Remark 2 A further refinement of the model in Eqn. (1) is obtained from the effect of the field on the coordinate $\zeta$ along the direction of propagation, where up to two $S=2$ mutually perpendicular polarizations propagate along $\zeta$. Consider two molecules with coordinates $r, \theta, \alpha, \zeta$ and $r, \theta, \alpha, \zeta^{\prime}$, with $\zeta^{\prime}>\zeta$ (i.e., the two molecules differ only in their locations along the direction of propagation); the molecule at $\zeta$ will see the field first while the other molecule will see the same field but starting at a later time shift of $\left(\zeta^{\prime}-\zeta\right) / c$, where $c$ is the speed of light. The Theorem ensures that all molecules will reach the target in the same time interval $t^{f}$ after initiation of the interaction with the laser field. In absolute terms, the time to reach the target is $t^{f}+\zeta / c$ for a molecule at location $\zeta$. Several comments are pertinent concerning the role of $\zeta$ :

$1 /$ If the target is a stationary state of the internal Hamiltonian $H_{0}$, then the effect of $\zeta$ is not an issue. In this case once a molecule reaches a target state it will remain there and "wait" for the other molecules to reach the same target value. A practical example of this behavior is controlled molecular fragmentation where the products fly off for detection.

$2 /$ If the probe pulse propagates collinearly with the control pulse, then $\zeta$ has no role regardless of whether the target is a stationary state or not, since the relative timing between the control and probe pulses is the same for any $\zeta$ value.

$3 /$ If the probe propagation direction is perpendicular to the control pulse and if the target state is not stationary, then there are two good operating conditions:

1. The relevant controlled process occurs over a short longitudinal distance such that there is essentially no $\zeta$ dependence in the dynamics. In this circumstance the Theorem still covers the transverse spatial effects and the molecular orientation effects.

2. The relevant controlled process occurs over an extensive longitudinal distance, and the probe can spatially resolve the dynamics along the $\zeta$ direction. In this case the probe could be tuned to take into account the local $\zeta$ dependence.

Under these various conditions the Theorem still applies. However, this general situation becomes more complex if a third polarization field $s=3$ is involved. A number of scenarios can be envisioned in this case along the same lines as discussed above. 
The very positive result reported in the Theorem has important consequences for the implementation of quantum control in the laboratory. The Theorem implies that the application of adaptive control techniques [5] will automatically seek out a single shaped optimal field $\epsilon^{s}(t), s=1, ., S$ for each polarization, working to sweep all of the molecules (quantum systems) to a common target regardless of (a) the spatial beam profile perpendicular to the direction of propagation, (b) the location of the molecules relative to the beam focal plane and (c) the orientation of the molecules. However, accompanying this collective control will generally also be a distribution of control mechanisms existing over the full sample. Molecules at distinct locations and orientations will likely follow different quantum control pathways, yet all collectively arriving at the same final desired state. In practice, the availability of only a finite pulse energy will ultimately limit the achieved control to lie in spatial regions and molecular orientations where $\cos \left(\alpha^{s}\right) u^{s}(R), s=1, ., S$ is greater than some small cutoff value $u_{c}$.

Bandwidth and other constraints on the pulse can also limit the attained control regardless of spatial or orientation effects. Most importantly, the Theorem implies that achieving control under spatially diverse conditions and random molecular orientations is not a matter of striking a compromise, but rather a task subject to optimization to reach the highest possible level of performance for all the molecules in the sample. This feature forms the foundation for attaining a practical level of signal intensity for feedback in the control experiments. Under the same conditions of the Theorem in this paper, it has recently been shown that the search for an optimal control field will not encounter any false extrema [10]. This latter result and the Theorem in this paper provide the primary basis to expect the success of future state preparation quantum control experiments, provided that sufficiently flexible control sources are available.

\section{Acknowledgments}

H.R. acknowledges support from the Department of Energy and G.T. acknowledges PICS CNRS-NSF and ANR C-QUID grants and support from INRIA Rocquencourt. 


\section{APPENDIX A: PROOF DETAIL ON THE EXISTENCE OF A PATH}

We proved that the Lie algebra generated by $i \mathcal{H}_{0}$ and $i \mathcal{B}^{s}$ contains all elements

$$
\left\{\operatorname{diag}\left(u_{1}^{s} S_{\xi}, \ldots, u_{M}^{s} S_{\xi}\right), \operatorname{diag}\left(u_{1}^{s} Y_{\xi}, \ldots, u_{M}^{s} Y_{\xi}\right) ; \mu_{\xi}^{s} \neq 0, s=1, ., S, \xi \in \Xi\right\}
$$

Consider now a path $i_{1}, \ldots, i_{L}$ in the graph $G$ (the vertices are not necessarily unique). Since at least one individual system is controllable, the graph $G$ is connected [19] ; thus, a path exists between any two vertices. But, the path may be formed from different dipoles $\mu^{s}$ : $\mu_{i_{j} i_{j+1}}^{s_{j}} \neq 0, j=1, \ldots, L-1$.

By iterating the commutators of these matrices in Eqn. (A1) one obtains that $\operatorname{diag}\left(u_{1}^{s_{1}} \ldots u_{1}^{s_{L-1}} S_{\left(i_{1}, i_{L}\right)}, \ldots, u_{M}^{s_{1}} \ldots u_{M}^{s_{L-1}} S_{\left(i_{1}, i_{L}\right)}\right)$ is in $\mathcal{L}$ too. We will show that the set of all such vectors $\left(u_{1}^{s_{1}} \ldots u_{1}^{s_{L-1}}, \ldots, u_{M}^{s_{1}} \ldots u_{M}^{s_{L-1}}\right)$ is of rank at least $M$, implying that all of the elements $\left(S_{\left(i_{1}, i_{L}\right)}, 0, \ldots, 0\right), \ldots,\left(0, \ldots, S_{\left(i_{1}, i_{L}\right)}, 0, \ldots, 0\right), \ldots,\left(0, \ldots, S_{\left(i_{1}, i_{L}\right)}\right)$ belong to the Lie algebra. Then we construct commutators of these elements and conclude as in [16] that the algebra spanned by $i \mathcal{H}_{0}$ and $i \mathcal{B}^{s}$ is maximal.

It is necessary to prove that under the hypothesis (3) or (4) for any $I, I^{\prime}$

$$
\operatorname{rank}\left\{\left(u_{1}^{s_{1}} \ldots u_{1}^{s_{L-1}}, \ldots, u_{M}^{s_{1}} \ldots u_{M}^{s_{L-1}}\right) ; \mu_{i_{j} i_{j+1}}^{s_{j}} \neq 0, L \geq 0, i_{1}=I, i_{L}=I^{\prime}\right\}>M
$$

Notice that for any $i \neq j, 1 \leq i, j \leq M$ under (3) or (4) there exists a $s \in\{1, ., S\}$ such that either $\left|u_{i}^{s}\right| \neq\left|u_{j}^{s}\right|$ or a cycle of odd length (i.e., involving an odd number of vertices) exists in the graph $G^{s}$ and $u_{i}^{s} \neq u_{j}^{s}$. In both situations a cycle of length $P_{i j}$ exists in the graph $G^{s}$ (thus in $G$ too) such that $\left(u_{i}^{s}\right)^{P_{i j}} \neq\left(u_{j}^{s}\right)^{P_{i j}}$. We now consider such cycles for each pair $(i, j)$. The cycle for $(i, j)$ can be repeated an arbitrary number of times if necessary. We may link cycles by paths in the connected graph $G$ : thus, a larger cycle $c_{1}, \ldots, c_{P}=c_{1}$ may be constructed in the graph $G$, with edge $c_{i} c_{i+1}$ from the subgraph $G^{s_{i}}$ such that the coefficients $k_{1}=u_{1}^{s_{1}} \ldots u_{1}^{s_{P-1}}, \ldots, k_{M}=u_{M}^{s_{1}} \ldots u_{M}^{s_{P-1}}$ are all different. We also used the following : if for some $i, j$ either $0 \neq b_{i} \neq b_{j} \neq 0$ or $0 \neq a_{i} \neq a_{j} \neq 0$ then $a_{i} b_{i}^{f} \neq a_{j} b_{j}^{f}$ except for possible only one value $f \in \mathbb{N}$.

Now for any couple $I, I^{\prime}$ as in Eqn. (A2) we can construct a path $i_{1}=I, \ldots, i_{R}=$ $I^{\prime}$ from $I$ to $I^{\prime}$ in $G$ that passes through $c_{1}$ at some intermediary step $i_{\alpha}$. We can insert the cycle $c_{1}, \ldots, c_{P}=c_{1}$ an arbitrary number of times into this path. Thus $\left\{\left(u_{1}^{s_{1}} \ldots u_{1}^{s_{L-1}}, \ldots, u_{M}^{s_{1}} \ldots u_{M}^{s_{L-1}}\right) ; \mu_{i_{j} i_{j+1}}^{s_{j}} \neq 0, L \geq 0, i_{1}=I, i_{L}=I^{\prime}\right\}$ will contain 
$\left(u_{1}^{s_{1}} \ldots u_{1}^{s_{\alpha-1}} k_{1}^{v} u_{1}^{s_{\alpha}} \ldots u_{1}^{s_{R-1}}, \ldots, u_{M}^{s_{1}} \ldots u_{M}^{s_{\alpha-1}} k_{M}^{v} u_{M}^{s_{\alpha}} \ldots u_{M}^{s_{R-1}}\right)=\left(k_{1}^{v} \prod_{\beta=1}^{R_{1}} u_{1}^{s_{\beta}}, \ldots, k_{M}^{v} \prod_{\beta=1}^{R_{M}} u_{M}^{s_{\beta}}\right)$ for any $v \in \mathbb{N}$. Since all $k_{m}, m=1, \ldots, M$ are different, we obtain Eqn. (A2).

[1] S. Shi, A. Woody, and H. Rabitz, J. Chem. Phys. 88, 6870 (1988).

[2] M. Shapiro and P. Brumer, Principles of the Quantum Control of Molecular Processes (WileyInterscience, Hokoken, N.J., 2003).

[3] S. Rice and M. Zhao, Optical Control of Quantum Dynamics (Wiley, 2000).

[4] R. Wu, H. Rabitz, G. Turinici, and I. Sola, Phys. Rev. A 70, 052507 (2004).

[5] R. S. Judson and H. Rabitz, Phys. Rev. Lett 68, 1500 (1992).

[6] A. Assion, T. Baumert, M. Bergt, T. Brixner, B. Kiefer, V. Seyfried, M. Strehle, and G. Gerber, Science 282, 919 (1998).

[7] T. Weinacht, J. Ahn, and P. Bucksbaum, Nature 397, 233 (1999).

[8] R. Bartels, S. Backus, E. Zeek, L. Misoguti, G. Vdovin, I. Christov, M. Murnane, and H. Kapteyn, Nature 406, 164 (2000).

[9] R. J. Levis, G. Menkir, and H. Rabitz, Science 292, 709 (2001).

[10] M. Rabitz, R. Hsieh and C. Rosenthal, Science 303, 1998 (2004).

[11] K. Sundermann, H. Rabitz, and R. de Vivie-Riedle, Phys. Rev. A 62, 013409 (2000).

[12] K. Sundermann and R. de Vivie-Riedle, Appl. Phys. B 71, 285 (2000).

[13] T. Brixner, G. Krampert, T. Pfeifer, R. Selle, G. Gerber, M. Wollenhaupt, O. Graefe, C. Horn, D. Liese, and T. Baumert, Phys. Rev. Lett. 92, 208301 (2004).

[14] G. Turinici, V. Ramakhrishna, B. Li, and H. Rabitz, J. Phys A: Math. and General 37, 273 (2003).

[15] B. Li, G. Turinici, V. Ramakhrishna, and H. Rabitz, J. Phys. Chem. B 106, 8125 (2002).

[16] G. Turinici and H. Rabitz, Phys. Rev. A 70, 063412 (2004).

[17] G. Turinici and H. Rabitz, Chem. Phys. 267, 1 (2001).

[18] V. Jurdjevic and H. Sussmann, J. Diff. Eq. 12, 313 (1972).

[19] G. Turinici and H. Rabitz, J. Phys.A. 36, 2565 (2003). 